Rabaska

Revue d'ethnologie de l'Amérique française

\title{
Germain Lemieux (1914-2008)
}

\section{Jean-Pierre Pichette}

Volume 6, 2008

URI : https://id.erudit.org/iderudit/019993ar

DOI : https://doi.org/10.7202/019993ar

Aller au sommaire du numéro

Éditeur(s)

Société québécoise d'ethnologie

ISSN

1703-7433 (imprimé)

1916-7350 (numérique)

Découvrir la revue

Citer ce document

Pichette, J.-P. (2008). Germain Lemieux (1914-2008). Rabaska, 6, 134-139.

https://doi.org/10.7202/019993ar d'utilisation que vous pouvez consulter en ligne.

https://apropos.erudit.org/fr/usagers/politique-dutilisation/ 


\section{Nécrologie}

\section{Germain Lemieux (1914-2008)}

Né à Cap-Chat, dans le comté de Gaspé au Québec, le 5 janvier 1914, le père Germain Lemieux, s.j., s'installe à Sudbury en 1941 afin d'enseigner les institutions grecques et latines au Collège du Sacré-Cœur (1941-1944, 1949-1953, 19561957), un établissement fondé par les jésuites. À l'exception de ses séjours d'études à Montréal, pour sa licence en théologie (19441948), et à Québec, pour sa maîtrise (1953$1955)$ et son doctorat (1959-1961) en études canadiennes, il passera la plus grande partie de sa carrière active en Ontario, étant tour à tour professeur d'histoire à la nouvelle Université de Sudbury (1957-1959) puis à la Laurentienne (1961-1965), ou de folklore, d'abord lors d'un bref passage à l'Université Laval (1965-1968) avant de revenir s'adonner pleinement à ses recherches en Ontario (1968-1975).

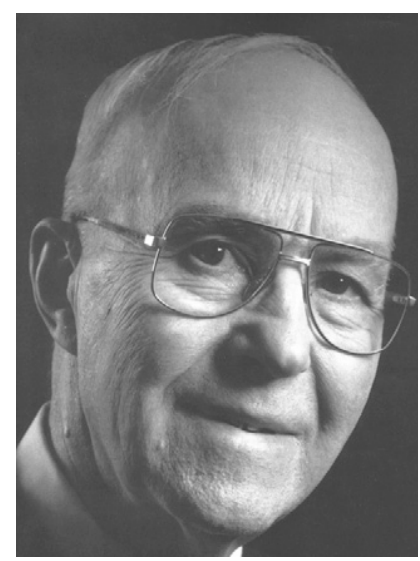

Germain Lemieux Gracieuseté du Centre francoontarien de folklore, Sudbury.

La série d'enquêtes folkloriques que le père Germain Lemieux avait inaugurées, le 12 septembre 1948, dans le nord de l'Ontario, allaient mener à la création, en 1960, d'un Institut de folklore à l'Université de Sudbury puis, après l'obtention d'une charte en 1972, du Centre franco-ontarien de folklore. On connaît bien aujourd'hui la suite : publication d'une cinquantaine de livres et brochures, dont la monumentale collection Les vieux m'ont conté. C'est pour mettre en valeur ce fonds documentaire que sera établi à l'Université de Sudbury, en 1975, un programme d'enseignement universitaire qui deviendra département de Folklore en 1981 puis, avec l'obtention d'un baccalauréat en 1995, département de Folklore et ethnologie de l'Amérique française ; on y dispense depuis des programmes complets de premier cycle en ethnologie, ce qui en fait le seul baccalauréat disponible à distance au Canada.

Les états de service du père Lemieux ont été remarqués tant au Canada qu'à l'étranger et la valeur de son entreprise reconnue par plusieurs grands prix et distinctions, notamment des doctorats honorifiques décernés par 
l'Université York, l'Université d'Ottawa et l'Université Laurentienne. En 1991, un colloque international lui était consacré - L'Euvre de Germain Lemieux, s.j. Bilan de l'ethnologie en Ontario français ${ }^{1}$-, ses publications faisant figure de référence à travers toute la francophonie, et même au-delà. Dans les annales franco-ontariennes, sa carrière, exceptionnelle tant par sa régularité que par sa durée, demeure un fait inattendu, et encore unique.

Ce jésuite chercheur, professeur, folkloriste, voire sculpteur qu'était le père Lemieux, aimait à se définir comme un touche-à-tout. Mais s'il fallait convenir d'une appellation pour bien cerner le personnage, ce serait sans doute «gardien de la mémoire franco-ontarienne» ou mieux «passeur de mémoire ». Tel est le titre que le Centre franco-ontarien de folklore attribua en 1998 à l'exposition, aujourd'hui itinérante, qui devait marquer le cinquantième anniversaire de son œuvre : «Passeur de mémoire en Ontario français ».

En août 2001, au terme d'un séjour de 60 ans à Sudbury, le père Germain Lemieux quittait définitivement ses fonctions de directeur de la recherche au Centre franco-ontarien de folklore et prenait sa véritable retraite à la maison des jésuites à Saint-Jérôme au Québec. C'est là qu'il est mort paisiblement, au bout de son âge, le 26 mars 2008.

\section{La chanson folklorique}

Soutenu par le père Lorenzo Cadieux, qui avait été son professeur à Gaspé et qui venait de fonder en 1942 la Société historique du Nouvel-Ontario, Germain Lemieux lançait en 1948 un premier «sondage » dans les paroisses des environs de Sudbury, soupçonnant que ces populations françaises avaient conservé une part de leur patrimoine oral. Ses enquêtes folkloriques allaient se poursuivre durant vingt-cinq ans et progressivement couvrir toute la région du nord de l'Ontario comprise entre Mattawa et Sault-Sainte-Marie.

Musicien, Germain Lemieux s'intéressera d'abord à la chanson folklorique et commencera assez tôt à présenter les premiers échantillons qu'il destine, en bénéficiaire reconnaissant, aux publications de la société historique qui le patronne. Un fascicule anonyme paraît en 1949, puis un second l'année suivante, portant son nom, Folklore franco-ontarien, Chansons [I] et $I I$, dans lesquels il propose à l'attention du public une quarantaine de chansons, paroles et musique, sélectionnées parmi ses cueillettes initiales. Ces brochures, saluées favorablement tant par la population et la presse que par plusieurs folkloristes du Canada français et de l'Europe, l'incitèrent à poursuivre ses recherches et à réunir une collection sonore de 3118 chansons qu'il a recueillies avec la technologie disponible : lourdes enregistreuses sur

1. Actes du colloque tenu à l'Université de Sudbury les 31 octobre, $1^{\text {er }}$ et 2 novembre 1991, sous la direction de J.-P. Pichette, Sudbury, Prise de parole et Centre franco-ontarien de folklore, 1993, 529 p. 
fil d'acier, sur ruban de papier puis de plastique, magnétophone plus léger et, ce qui serait une primeur mondiale, magnétoscope portatif dès 1969 .

En 1963, Germain Lemieux tient la chronique "Folklore » de l'hebdomadaire L'Information du diocèse de Sault-Sainte-Marie et il livre, en 38 courts articles, une sorte d'introduction à la chanson populaire. Il en refond le contenu l'année suivante chez le même éditeur : cette brochure, intitulée Chanteurs franco-ontariens et leurs chansons, fournit une description des fonctions, des thèmes et des motifs des chansons recueillies et conservées dans les collections du centre de folklore de Sudbury. Dix ans plus tard, le père Lemieux prépara une anthologie en deux volumes, Chansonnier francoontarien 1 et 2, sorte de "romancéro franco-ontarien » composé de 112 chansons de sa collection. En 1986, un éditeur montréalais réédita cette anthologie en trois fascicules, sous le titre Les vieux m'ont chanté.

\section{Le conte populaire}

C'est à l'école de Luc Lacourcière que le père Germain Lemieux s'intéressera au conte populaire. Inscrit au programme de maîtrise de l'Université Laval en 1953, il prépare, sous la direction de son maître, l'étude comparative d'un conte hagiographique : Placide-Eustache (Aarne-Thompson 938). Cette même année, il publie une brochure, Contes populaires franco-ontariens, puis une seconde en 1958, contenant la version remaniée de cinq contes populaires recueillis dans la région de Sudbury selon la méthode ethnographique. Ces deux plaquettes annoncent, à n'en pas douter avec le recul du temps, la vaste entreprise de diffusion à laquelle il vouera vingt ans de sa vie à compter de 1973.

Entre temps, il aura terminé sa thèse de maîtrise en 1955 puis entrepris et soutenu en 1961 une thèse de doctorat sur le même sujet. L'ouvrage paraîtra aux Presses de l'Université Laval en 1970 dans la collection « Les Archives de folklore » sous le titre Placide-Eustache. Sources et parallèles du contetype 938 .

\section{L'intention pédagogique}

Mais Germain Lemieux ne perdra jamais sa motivation initiale qui était avant tout d'ordre pédagogique. En effet, elle résultait d'une préoccupation personnelle de ce professeur du Collège du Sacré-Cœur de Sudbury qui avait d'abord découvert des correspondances entre les récits de la mythologie grecque qu'il enseignait et les contes populaires qu'il avait entendus dans son enfance à Cap-Chat en Gaspésie. Il chercha constamment à sensibiliser ses élèves à la présence de ces mythes dans leur culture et même ses étudiants lors de son passage à l'Université de Sudbury en 1958. 
Ce souci pédagogique explique en outre la plus grande partie de son œuvre, tant ses articles de vulgarisation que son œuvre maîtresse. Comme on l'a vu pour la chanson, il entend démontrer, dans une série d'articles parus en 1961 dans Le Droit d'Ottawa, que les conteurs analphabètes sont les héritiers des Orientaux et il s'emploie à dépister les sources orientales dans la tradition orale franco-ontarienne. Il poursuivra cette démonstration dans l'hebdomadaire L'Information en 1963 et 1964 puis reprendra cette matière, enrichie par son enseignement universitaire, dans la brochure De Sumer au Canada français, sur les ailes de la tradition en 1968.

\section{L'œuvre maîtresse}

La modification du statut juridique de l'Institut de folklore, qui se mua en Centre franco-ontarien de folklore durant l'année 1972, sonnait l'heure d'un nouveau départ. Cette même année, paraissait en coédition Les Jongleurs du billochet dont le sous-titre précise : Conteurs et contes franco-ontariens. Véritable introduction à l'œuvre gigantesque qui va suivre, ce livre éclaire à la fois le milieu humain, les conditions matérielles et la technique préconisée par l'auteur dans ses enquêtes en Ontario français, et il traite en outre de la personnalité des principaux conteurs décédés avant 1972.

En 1973, Germain Lemieux entreprenait la diffusion de sa pièce de résistance, Les vieux m'ont conté, une coédition des maisons Bellarmin de Montréal et Maisonneuve et Larose de Paris. En 32 volumes, publiés à un rythme plus ou moins semestriel jusqu'en 1991, les éditeurs ont livré au public plus de 10000 pages, soit l'intégrale des 646 contes et légendes recueillis et annotés par Germain Lemieux, avec l'aide de quelques collaborateurs, entre 1953 et 1980. Aux deux tiers des contes de provenance franco-ontarienne, s'ajoutent aussi des récits du Nouveau-Brunswick, du Québec et du Manitoba. Les textes y apparaissent en deux versions : la version remaniée, à laquelle renvoie un index analytique, et la version originale, avec la fiche technique du document, à laquelle correspond un lexique des mots populaires.

Cette œuvre imposante a déjà profondément marqué la discipline ethnologique du Canada français : des comptes rendus par dizaines ont applaudi, commenté et critiqué la parution de ces répertoires et une quinzaine d'articles, écrits par des chercheurs de plusieurs disciplines, n'ont pas manqué de les analyser. Mais ce n'est pas principalement pour eux que l'auteur les a rassemblés. Dans la présentation du premier tome, le père Lemieux poursuivait un autre objectif : « Notre but est d'abord, redisons-le, de faire connaître notre littérature orale à un plus grand public, de façon à rejoindre, un jour ou l'autre, un artiste qui se laisse gagner par la poésie de ces récits ${ }^{2} »$.

2. G. Lemieux, Les vieux m'ont conté, vol. 1, p. 20. 
C'est pourquoi il permit qu'on en tirât des adaptations littéraires et artistiques et il y collabora souvent lui-même. Ainsi sont nés une demidouzaine de livres et brochures dont Les vieux m'ont conté 1 et 2 en format de poche et version remaniée, Contes de mon pays et Ti-Jean et le gros roi de Serge Wilson et Claude Poirier. Ces deux derniers ont produit cinq albums de bandes dessinées en grand et en petit format dans la collection « Contes de mon pays » aux éditions Héritage. Enfin, l'artiste Claire GuillemetteLamirande publiait en 1979 Contes et couleurs de l'Ontario français, reproduction d'une série de neuf aquarelles illustrant des contes du père Lemieux.

\section{Remarque finale}

Le père Germain Lemieux a été l'animateur privilégié de la tradition orale en Ontario français, puisqu'il a écrit, dirigé et généré une centaine de livres, brochures, albums et articles de toute nature sur les genres auxquels il porta attention, la chanson et le conte principalement ${ }^{3}$.

Aujourd'hui l'œuvre du père Lemieux se poursuit par les institutions que son travail a créées. D'abord, le Centre franco-ontarien de folklore qui s'est donné comme mission de mettre en valeur, pour le bénéfice de la population ontarienne, le folklore et le patrimoine. C'est là qu'on conserve la collection complète que ce chercheur a documentée au cours de sa carrière. À la veille du soixantième anniversaire de la première enquête du père Lemieux (1948) et trente-cinq ans après son incorporation (1972), ce centre est devenu le chef de file dans le domaine de la conservation et de l'animation du patrimoine. Les travaux pionniers de son fondateur et la réalisation du vaste Inventaire du patrimoine franco-ontarien lui ont acquis une renommée enviable. En 1991, le ministère ontarien de la Culture, du tourisme et des loisirs reconnaissait le Centre comme organisme provincial à vocation patrimoniale. De même, le prix Parcs Canada, qui lui a été remis par le ministère du Patrimoine canadien en 1996, soulignait sa contribution remarquable à la conservation et à la mise en valeur de ce patrimoine.

Pour sa part, le département de Folklore et ethnologie de l'Amérique française de l'Université de Sudbury se charge des objectifs d'ordre scientifique du projet du père Lemieux. Et il le fait par la formation des étudiants que le domaine du patrimoine intéresse. Depuis 1981, sous la direction de leurs professeurs, ceux-ci ont relancé les enquêtes sur le terrain un peu partout en Ontario dans le cadre de certains cours, à titre de recherche et de travaux pratiques. En vingt-cinq ans, ils ont accumulé des milliers de documents ethnographiques qui seront traités dans le laboratoire que le

3. J.-P. Pichette, Répertoire ethnologique de l'Ontario français. Guide bibliographique et inventaire archivistique du folklore franco-ontarien, Ottawa, Les Presses de l'Université d'Ottawa, 1992, x-230 p. 
département est à mettre en place.

Voilà comment l'œuvre d'un seul homme aura pu inspirer d'autres artisans et se perpétuer durant plusieurs générations.

Jean-Pierre Pichette

Université Sainte-Anne, Pointe-de-l’Église

\section{Bibliographie de Germain Lemieux}

1949-1950 Folklore franco-ontarien : chansons I et II, Sudbury, La Société historique du Nouvel-Ontario [SHNO], « Documents historiques » 17, 1949, 48 p., et « Documents historiques » 20, 1950, 48 p.

1953-1958 Contes populaires franco-ontariens [I] et II, Sudbury, Shno, « Documents historiques » 25, 1953, 40 p., et « Documents historiques » $35,1958,60 \mathrm{p}$.

1963-1964 Chanteurs franco-ontariens et leurs chansons, Sudbury, SHNo, « Documents historiques » 44-45, 1963-1964, 113 p.

1968 De Sumer au Canada français sur les ailes de la tradition, Sudbury, SHNo, « Documents historiques » 51-52, 1968, 73 p.

1970 Placide-Eustache: sources et parallèles du conte-type 938, Québec, PuL, «Archives de folklore »10, 1970, VIII-214 p.

1972 Les Jongleurs du billochet : conteurs et contes franco-ontariens, Sudbury, SHNO, « Documents historiques » 61-62-63, Paris, Maisonneuve et Larose, et Montréal, Bellarmin, 1972, 134 p.

1973-1993 Les vieux m'ont conté, Montréal, Bellarmin, et Paris, Maisonneuve et Larose, 1973-1993, 33 tomes.

1974-1975 Chansonnier franco-ontarien I et II, Sudbury, SHNO, « Documents historiques » 64, 1974, [4]-138 p., et « Documents historiques »66, 1975, [4]-142 p.

1978 Contes de mon pays, Montréal, Héritage, « Katimavik », [1978], c1976, 159 p.

1981 Les vieux m'ont conté, Montréal, Bellarmin, et Laval, Éditions FM, 1981, 2 tomes.

1981 Le Four de glaise, Sudbury, Prise de parole, et Laval, Éditions FM, 1981, $58 \mathrm{p}$.

1982 La Vie paysanne 1860-1900, Sudbury, Prise de parole, et Laval, Éditions FM, 1982, 239 p.

1986 Les vieux m'ont chanté, Laval, Éditions FM, 1986, 3 tomes. 